\title{
Enzyme Kinetics, Structural Analysis and Molecular Modeling Studies on a Series of Schistosoma mansoni PNP Inhibitors
}

\author{
Matheus P. Postigo, ${ }^{a}$ Renata Krogh, ${ }^{a}$ Marcela F. Terni, ${ }^{a}$ Humberto M. Pereira, ${ }^{a}$ \\ Glaucius Oliva, ${ }^{a}$ Marcelo S. Castilho ${ }^{*, b}$ and Adriano D. Andricopulo ${ }^{*, a}$
}

\author{
${ }^{a}$ Laboratório de Química Medicinal e Computacional, Instituto de Física de São Carlos, \\ Universidade de São Paulo, 13560-970 São Carlos-SP, Brazil \\ ${ }^{b}$ Laboratório de Bioinformática e Modelagem Molecular da Faculdade de Farmácia, \\ Universidade Federal da Bahia,40170-290 Salvador-BA, Brazil
}

\begin{abstract}
A enzima purina nucleosídeo fosforilase do parasita Schistosoma mansoni (SmPNP) é um alvo molecular atrativo para o desenvolvimento de candidatos a novos fármacos para o tratamento da esquistossomose, doença tropical negligenciada que afeta mais de 200 milhões de pessoas em todo mundo. No presente trabalho, estudos de cinética enzimática foram conduzidos para a determinação da potência e do mecanismo de inibição de uma série de inibidores da enzima SmPNP. Além das investigações bioquímicas, estudos cristalográficos e de modelagem molecular revelaram importantes bases moleculares para a afinidade de ligação frente à enzima alvo, levando ao desenvolvimento de relações entre a estrutura e atividade (SAR).
\end{abstract}

The enzyme purine nucleoside phosphorylase from Schistosoma mansoni (SmPNP) is an attractive molecular target for the development of novel drugs against schistosomiasis, a neglected tropical disease that affects about 200 million people worldwide. In the present work, enzyme kinetic studies were carried out in order to determine the potency and mechanism of inhibition of a series of SmPNP inhibitors. In addition to the biochemical investigations, crystallographic and molecular modeling studies revealed important molecular features for binding affinity towards the target enzyme, leading to the development of structure-activity relationships (SAR).

Keywords: neglected tropical diseases, schistosomiasis, enzyme inhibition, crystal structure, binding affinity

\section{Introduction}

Neglected tropical diseases are responsible for millions of deaths and disabilities every year. Schistosomiasis is a major cause of illness, morbidity and death worldwide. Currently, there are about 200 million people infected, with more than 650 million living in endemic areas. ${ }^{1}$ Praziquantel is the only effective drug available for the treatment of schistosomiasis, however, less than $20 \%$ of those in need currently receive the drug. Additionally, praziquantel has been in use for more than 20 years and significant resistance to the chemotherapy has emerged. ${ }^{2}$ Therefore, this scenario highlights the urgent need for the development of new drugs to treat the disease., ${ }^{2,3}$

*e-mail: aandrico@ifsc.usp.br, castilho@ufba.br
Enzymes are attractive biological targets for smallmolecule drug discovery. ${ }^{4,5}$ Purine nucleoside phosphorylase (PNP, EC 2.4.2.1) is a key enzyme in the purine salvage pathway which has been primarily studied as a target for the treatment of T-cell proliferative diseases, such as T-cell leukemias or lymphomas, organ transplant rejection, rheumatoid arthritis, psoriasis, and some other autoimmune diseases. ${ }^{6,7}$ In fact, two inhibitors discovered at BioCryst Pharmaceuticals are currently undergoing clinical trials: (i) BCX1777 (forodesine) for the treatment of cutaneous T-cell lymphoma, chronic lymphocytic leukemia and acute lymphoblastic leukemia; and (ii) BCX4208 for the treatment of gout. ${ }^{8}$ More recently, it has been suggested that PNP inhibitors could also be used for the therapy of parasitic tropical diseases, such as malaria and schistosomiasis. ${ }^{9-14}$ The parasite Schistosoma mansoni, one of the etiologic agents of human schistosomiasis, lacks 
the de novo pathway for purine biosynthesis and depends entirely on the salvage pathway for its purine requirements for synthesis of RNA and DNA., ${ }^{711,13}$ Taking into account that PNP from S. mansoni (SmPNP) is an important component of the purine salvage pathway and has been identified as an attractive drug target, the use of selective inhibitors can cause purine starvation, leading to death of the parasite. ${ }^{7,13,14}$

In the present work, we have evaluated a series of guanine derivatives against SmPNP in order to determine their in vitro potency, affinity and mechanism of inhibition. In addition, crystallographic and molecular modeling studies were performed to identify the interaction profile of the inhibitors, as well as key structural elements responsible for the experimental properties.

\section{Experimental}

\section{SmPNP, data set and biochemical assays}

SmPNP was expressed and purified as described previously., ${ }^{911}$ Xanthine oxidase was of the best grade available from Sigma Aldrich and was used without further purification. All other reagents and solvents were obtained commercially from Sigma Aldrich and were of the highest purity available. The data set of inhibitors (1-20, Table 1) employed in this work, consisting of a series of guanine, 9-substituted-guanine, 9-substituted-9-deazaguanine and 8,9-disubstituted-9-deazaguanine derivatives, were synthesized by scientists at BioCryst Pharmaceuticals Inc. and are the gift of that organization. Kinetic measurements were carried out spectrophotometrically with the aid of a Cary100 UV-Vis spectrophotometer, using a standard coupled assay as previously described. ${ }^{13-16}$ The reaction mixture contained $5 \mathrm{nmol} \mathrm{L}^{-1} \operatorname{SmPNP}$ (as the monomer), $50 \mathrm{mmol} \mathrm{L}^{-1}$ phosphate buffer $\left(\mathrm{KPO}_{4}, \mathrm{pH} 7.4\right)$, $10 \mu \mathrm{mol} \mathrm{L}^{-1}$ inosine, and xanthine oxidase 40 milliunits $\mathrm{mL}^{-1}$. Uric acid formation was monitored at $293 \mathrm{~nm}$, in triplicate at $25{ }^{\circ} \mathrm{C}$ (extinction coefficient for uric acid, $\left.\varepsilon_{293}=12.9 \mathrm{~L} \mathrm{mmol}^{-1} \mathrm{~cm}^{-1}\right) .{ }^{17}$ The percentage of inhibition was calculated according to the following equation:

$\%$ of Inhibition $=100 \times\left(1-\mathrm{V}_{\mathrm{i}} / \mathrm{V}_{0}\right)$

where, $\mathrm{V}_{\mathrm{i}}$ and $\mathrm{V}_{0}$ are the initial velocities (enzyme activities) determined in the presence and in the absence of inhibitor, respectively. Compound 3, a known SmPNP inhibitor, was used as a positive control for enzyme inhibition. ${ }^{13}$ Values of $\mathrm{IC}_{50}$ (concentration of compound required for $50 \%$ inhibition of $S m \mathrm{PNP}$ ) for the whole series of inhibitors were independently determined by making rate measurements for at least six inhibitor concentrations. The type of inhibition and $K_{\mathrm{i}}$ (inhibitor constant) values were determined for a subset of potent inhibitors under the same experimental conditions for three different inhibitor concentrations at five varying substrate concentrations $\left(5.0,7.5,10,15\right.$ and $\left.20 \mu \mathrm{mol} \mathrm{L}^{-1}\right)$. All kinetic parameters were determined from the collected data by nonlinear regression employing the SigmaPlot enzyme kinetics module. The values represent means of at least three individual experiments. Values of $\mathrm{IC}_{50}$ for inhibitors 3, 7, 13, 18 and 20 measured at $10 \mu \mathrm{mol} \mathrm{L}^{-1}$ inosine are in good agreement with those previously described, ${ }^{13,14}$ whereas comparable values are not available for the other inhibitors of the data set.

\section{Molecular modeling studies}

The analyses, calculations and visualizations were performed using the programs SYBYL 8.0 (Tripos Inc., St. Louis, USA) and Pymol 0.99 (DeLano Scientific, Palo Alto, USA). Molecular docking and scoring protocols, as implemented in FlexX (BioSolveIT GmbH, Sankt Augustin, Germany) and GOLD 4.12 (Cambridge Crystallographic Data Centre, Cambridge, U.K.), were employed to search for reasonable binding poses of the ligands within the SmPNP binding pocket. The 3D structures of all SmPNP inhibitors used in the modeling studies were constructed using CONCORD and standard geometric parameters of the molecular modeling software package SYBYL 8.0 running on Red Hat Enterprise Linux workstations. Although GOLD assigns atoms potential according to a pre-established internal function, all 3D structures were energetically minimized through atom-centered partial charge AM1ESP calculations as implemented in MOPAC 6.0 before docking runs. This strategy aimed at avoiding possible bond length or bond angles distortions present in any molecule. The X-ray crystallographic coordinates for SmPNP in complex with acetate used in the docking protocols were retrieved from the PDB databank (PDB ID 1TD1, $1.90 \AA$ resolution). Then, ligands and water molecules were discarded and the hydrogen atoms were added in standard geometry using the Biopolymer module as implemented in SYBYL 8.0. Histidine, glutamine, and asparagine residues in the binding site were manually checked for possible flipped orientation, protonation, and tautomeric states with Pymol 0.99 side-chain wizard script. Considering that the kinetic assays were carried out in pH 7.4 in this work, Glu203 side-chain was considered as fully ionized in all docking runs. The search space sampled in docking runs consists 
of all amino acid residues within a sphere radius of $6.5 \AA$ (FlexX) or 10.0 $\mathrm{A}$ (GOLD) centered on the oxygen atom of Tyr202. Default parameters were employed to generate the poses, which were then ranked by FlexX and GOLDSCORE scoring functions. Next, the best ranked conformation of each ligand was submitted to the web-based interface DrugScore ONLINE to rescore the proposed binding modes. ${ }^{18}$ Only poses that rank well in both function and that were consistent with kinetic studies were considered further.

\section{Crystallization and soaking of SmPNP}

The highly purified $S m P N P$ enzyme was crystallized as previously described. ${ }^{11-13} \mathrm{Next}$, crystals grown at $4{ }^{\circ} \mathrm{C}$ in $20 \%$ PEG 1500, $15 \mathrm{mmol} \mathrm{L}^{-1}$ sodium acetate buffer ( $\mathrm{pH} 4.9$ or 5.0) and $20 \%$ glycerol (buffer A) were soaked for 48$96 \mathrm{~h}$ in solutions containing $10 \mathrm{mmol} \mathrm{L}^{-1}$ of compound 7 (Table 1), dissolved in 1:9 DMSO/buffer A.

\section{$X$-ray data collection and structure refinement}

$\mathrm{X}$-ray diffraction data were collected at cryogenic temperatures $(100 \mathrm{~K})$ at W01B-MX-2 beamline, equipped with a Marmosaic 225 CCD scanner, of the LNLS (National Laboratory of Synchrotron Light, in Campinas - Brazil). The crystals of SmPNP in complex with compound 7 (SmPNP-7) diffracted up to $2.30 \AA$. The data were indexed and integrated using the program MOSFLM and scaled using the program SCALA from the CCP4 suite. ${ }^{19-22}$ Next, the starting phases were determined by molecular replacement using SmPNP in complex with acetate (PDB ID 1TD1) as the search model, as available in the program Molrep. ${ }^{21}$ The model refinement was carried out with Refmac ${ }^{22}$ and PHENIX ${ }^{23}$ using $\sigma_{\mathrm{a}}$-weighted 2Fo-Fc and $\mathrm{Fo}-\mathrm{Fc}$ electron density maps. The compounds were automatically placed in the electron density using the "Find Ligand" routine of Coot. ${ }^{24}$ Additional manual building and placement of water molecules were performed with the Coot and PHENIX software. During all steps of the refinement, $5 \%$ of the data were used to calculate the free R-factor ${ }^{25}$ and thus avoid overfitting. All final models were validated using PROCHECK. ${ }^{26}$ The coordinates and structures factors of SmPNP-7 complex are deposited in the PDB under ID 3DJF.

\section{Results and Discussion}

Considering the key role of PNP in the purine salvage pathway of the $S$. mansoni parasite, this enzyme has been selected as a potential target for the chemotherapeutic treatment of schistosomiasis. Despite the significant amount of structure-activity relationship (SAR) studies available for inhibitors of the mammalian PNPs, ${ }^{15,27}$ similar investigations for SmPNP remain outstanding, and would be essentially important for the design of new inhibitors. The difference of about 6-fold in the $K_{\mathrm{M}}$ (MichaelisMenten constant) values between human and parasite PNPs (human PNP $=41 \mu \mathrm{mol} \mathrm{L}^{-1}, S m \mathrm{PNP}=6.4 \mu \mathrm{mol} \mathrm{L}^{-1}$ ) for the natural substrate (inosine) indicates that binding affinity depends on specific molecular interactions between smallmolecule ligand and protein. As a matter of fact, the first $S m$ PNP inhibitors were only recently characterized in the literature. ${ }^{13,14}$ In an effort to expand our studies, enzyme kinetic, crystallographic and molecular modeling studies were carried out to investigate the fundamental chemical and structural requirements involved in the inhibition of the catalytic activity of $S m$ PNP by a series of guanine derivatives. In this context, a series of twenty compounds (Table 1) was experimentally evaluated to determine their in vitro potency $\left(\mathrm{IC}_{50}\right)$ and to provide new SAR information.

As can be seen, the two guanine derivatives of the data set (compounds $\mathbf{1}$ and 2) showed moderate inhibitory potency against $\operatorname{SmPNP}\left(\mathrm{IC}_{50}\right.$ values of 39 and $23 \mu \mathrm{M}$, respectively). These results contrast with that of compound $\mathbf{3}\left(\mathrm{IC}_{50}=1.35 \mu \mathrm{M}\right)$, a 9-benzyl-9-deazaguanine derivative, which is approximately 30 -fold more potent than the structurally related guanine analog $\mathbf{2}$. This confirms previous studies showing that 9-deazaguanine analogs are more potent than the corresponding guanine analogs. ${ }^{6,28,29}$ A hypoxanthine analog of the data set (compound 4, $\left.\mathrm{IC}_{50}=17 \mu \mathrm{M}\right)$, which is structurally related to compounds $\mathbf{2}$ and $\mathbf{3}$, is $\mathbf{1 2}$-fold less potent than compound $\mathbf{3}$, and about 2 -fold more potent than the guanine analog 2 . These results highlight the importance of the 9-deazaguanine scaffold in the development of selective inhibitors of SmPNP, indicating that stereochemical features in the ribose binding site might play a pivotal role in SmPNP inhibition.

In order to extend our SAR studies, we have evaluated some 9-deazaguanines (5-20) possessing a variety of substituents in the 9-position. On the basis of structure 3, we investigated the bioisosteric replacement of the methylene group (benzyl group) at position 9 of the purine scaffold with an oxygen atom, leading to compound $\mathbf{5}$. However, this structural modification led a 3 -fold decrease in potency. The substitution of the hydrogen at position 8 of the purine ring with a methyl group afforded a very weak inhibitor of $\operatorname{SmPNP}$ (compound $\mathbf{6}, \mathrm{IC}_{50}=200 \mu \mathrm{M}$ ). On the other hand, the presence of heteroaromatic moieties at position 9 of the purine ring ( 7 and 8 ) in place of the traditional phenyl group improved potency against SmPNP, with $\mathrm{IC}_{50}$ values in the nanomolar range (820 
586

Enzyme Kinetics, Structural Analysis and Molecular Modeling Studies on a Series of Schistosome mansoni J. Braz. Chem. Soc.

Table 1. Inhibitory potency of a series of SmPNP inhibitors

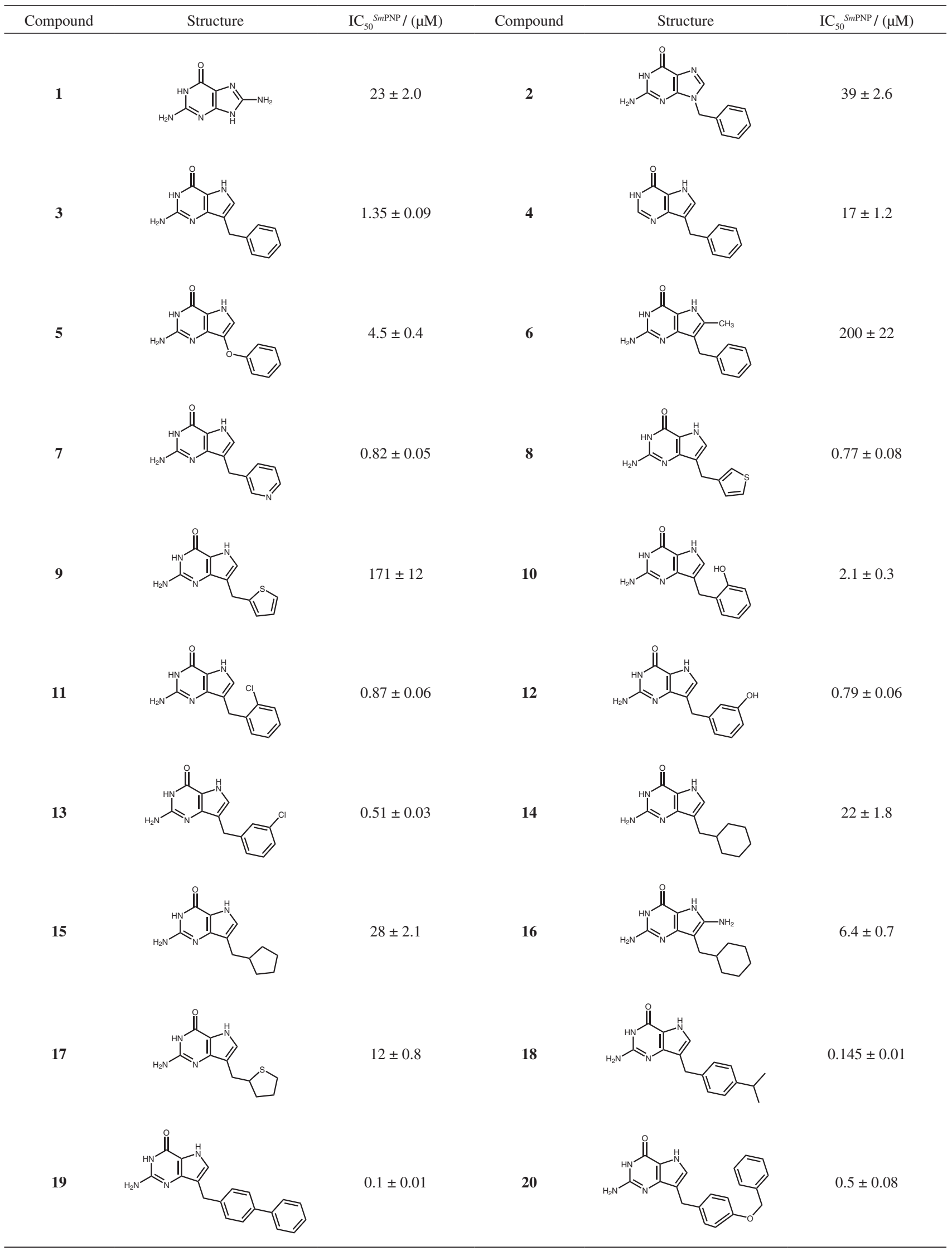


and $770 \mathrm{nM}$, respectively). Our SAR data show that the replacement of aromatic (phenyl, pyridine, thiophene) to non-aromatic moieties led to new compounds with decreased inhibitory potency $\left(\mathbf{1 4}, \mathrm{IC}_{50}=22 \mu \mathrm{M} ; \mathbf{1 5}\right.$, $\mathrm{IC}_{50}=28 \mu \mathrm{M} ; \mathbf{1 6}, \mathrm{IC}_{50}=6.4 \mu \mathrm{M}$; and 17, $\mathrm{IC}_{50}=12 \mu \mathrm{M}$ ) when compared with those of compounds $\mathbf{7}$ and $\mathbf{8}$. The substitution on the phenyl group of compound $\mathbf{3}$ was also considered, employing a series of 9-deazaguanines having the phenyl group substituted by hydrophilic (10, $\mathrm{IC}_{50}=2.1 \mu \mathrm{M}$ and $\left.\mathbf{1 2}, \mathrm{IC}_{50}=0.79 \mu \mathrm{M}\right)$ and hydrophobic (11, $\mathrm{IC}_{50}=0.87 \mu \mathrm{M}$ and $13, \mathrm{IC}_{50}=0.51 \mu \mathrm{M}$ ) substituents. As can be seen in Table 1, the presence of the ortho- or meta-chlorine (11 or 13) or meta-hydroxyl (12) led to a slight increase in potency, which was not observed for compound $10\left(\mathrm{IC}_{50}=2.1 \mu \mathrm{M}\right)$. On the other hand, substituents in the para position of the phenyl ring led to the most potent inhibitors of this series, compounds $18\left(\mathrm{IC}_{50}=0.14 \mu \mathrm{M}\right)$ and $\mathbf{1 9}\left(\mathrm{IC}_{50}=0.10 \mu \mathrm{M}\right)$, suggesting that bulkier hydrophobic groups would be important for biological activity. The other para-substituted inhibitor of the series also confirms this tendency (compound 20, $\left.\mathrm{IC}_{50}=0.5 \mu \mathrm{M}\right)$.

A

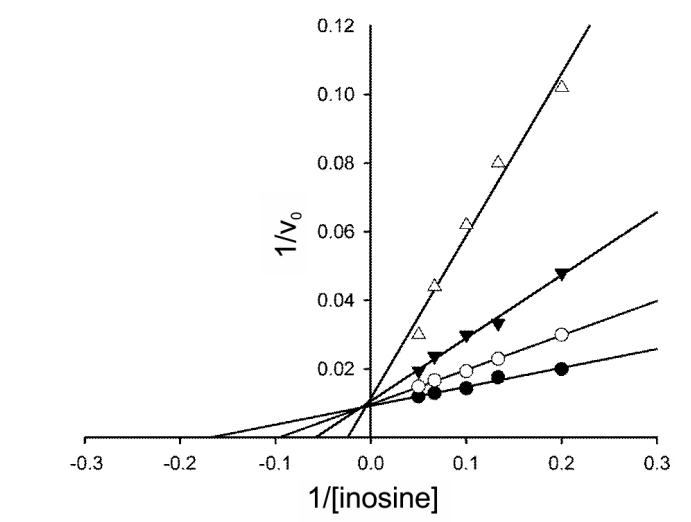

C

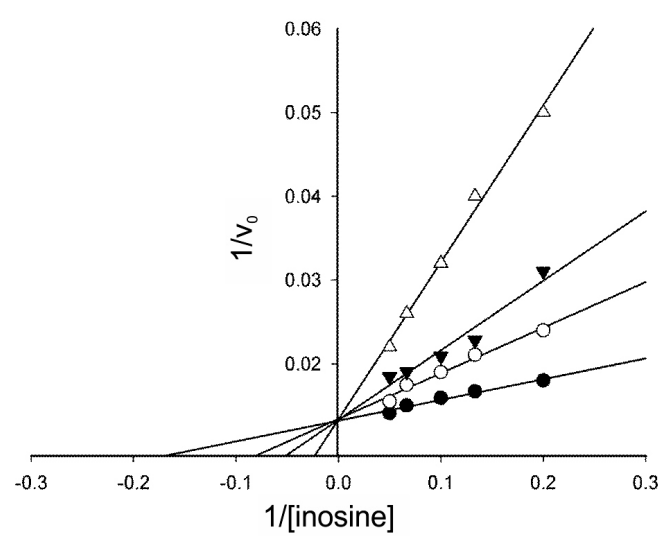

Although the SAR information gathered from this series is valuable and revealed several structural aspects related to the inhibitory potency, the underlying explanation for these results would require more in-depth investigation. Two things are especially important. First, the evaluation of the mechanism of SmPNP inhibition associated to the determination of the affinity of the inhibitors. Second, the structural analysis of the binding mode of the inhibitors into the $S m$ PNP active site. In order to explore the mechanism of inhibition in more detail, $K_{\mathrm{i}}$ values and the type of inhibition with respect to the physiological substrate were determined employing a subset of inhibitors as shown in Table 2 and Figures 1 and 2. The Lineweaver-Burk double-reciprocal plots show the intercepts of the lines

Table 2. $K_{\mathrm{i}}$ values for a subset of potent $S m$ PNP inhibitors

\begin{tabular}{lcc}
\hline Compound & $K_{\mathrm{i}} /(\mu \mathrm{M})$ & $\mathrm{IC}_{50} / K_{\mathrm{i}}$ \\
\hline $\mathbf{7}$ & 0.451 & 1.8 \\
$\mathbf{1 3}$ & 0.120 & 4.2 \\
$\mathbf{1 8}$ & 0.038 & 3.9 \\
$\mathbf{1 9}$ & 0.071 & 1.4 \\
\hline
\end{tabular}

B

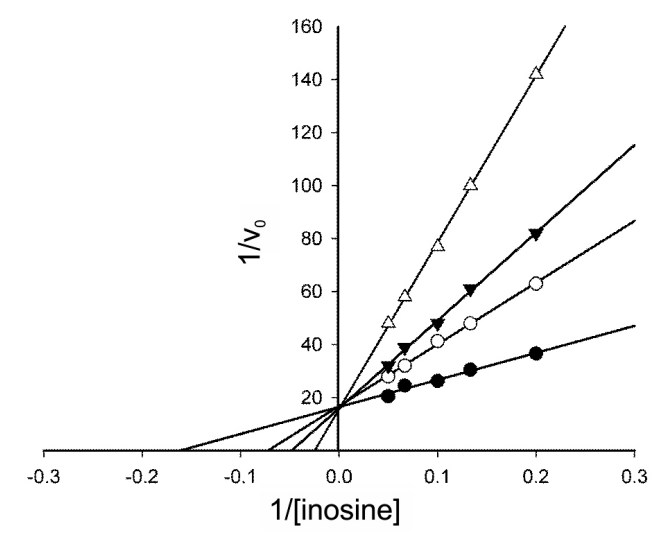

D

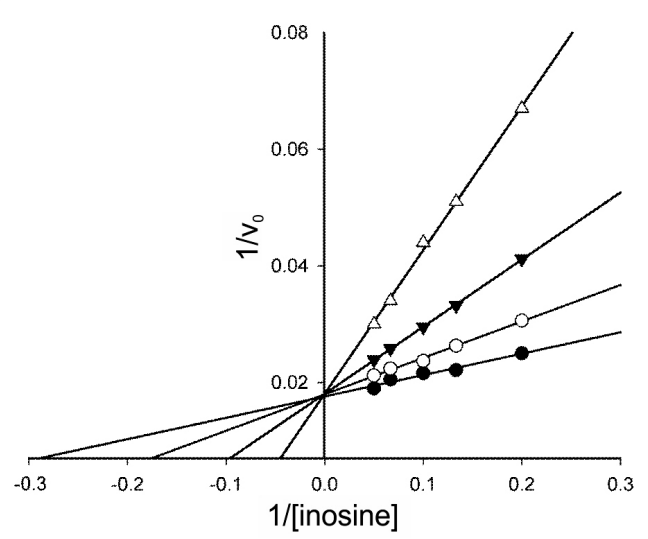

Figure 1. Competitive inhibitory profile of the $S m$ PNP inhibitors 7 (A), 13 (B), 18 (C) and $\mathbf{1 9}$ (D). Kinetic data was collected in the presence of increasing concentrations of inhibitor. Panel A $0.8 \mu \mathrm{mol} \mathrm{L}^{-1}(\bigcirc), 1.6 \mu \mathrm{mol} \mathrm{L}^{-1}(\boldsymbol{\nabla}), 3.0 \mu \mathrm{mol} \mathrm{L}-1(\triangle)$. Panel B $0.8 \mu \mathrm{mol} \mathrm{L}^{-1}(\bigcirc), 1.6 \mu \mathrm{mol} \mathrm{L}^{-1}(\boldsymbol{\nabla}), 3.0 \mu \mathrm{mol} \mathrm{L}^{-1}(\triangle)$. Panel C $150 \mathrm{nmol} \mathrm{L}^{-1}(\bigcirc), 300 \mathrm{nmol} \mathrm{L}^{-1}(\boldsymbol{\nabla})$ and $600 \mathrm{nmol} \mathrm{L}^{-1}(\triangle)$. Panel D $200 \mathrm{nmol} \mathrm{L}^{-1}(\bigcirc), 400 \mathrm{nmol} \mathrm{L}^{-1}(\boldsymbol{\nabla})$ and $800 \mathrm{nmol} \mathrm{L}^{-1}(\triangle)$. In all panels the absence of inhibitor is depicted by $(\bullet)$. 
A

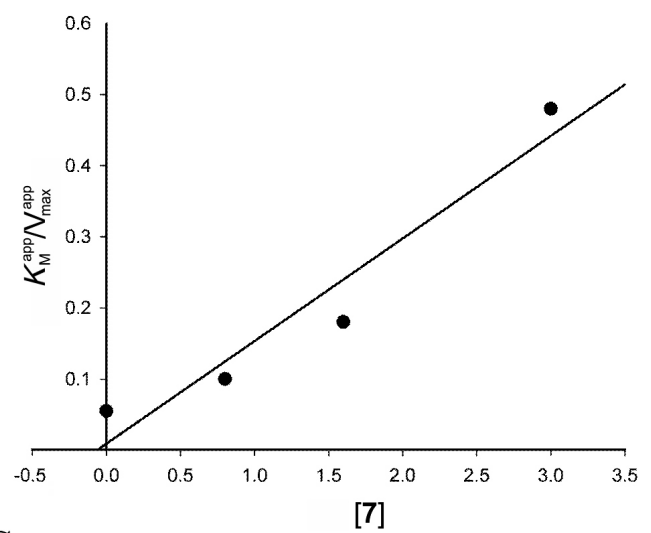

$\mathrm{C}$

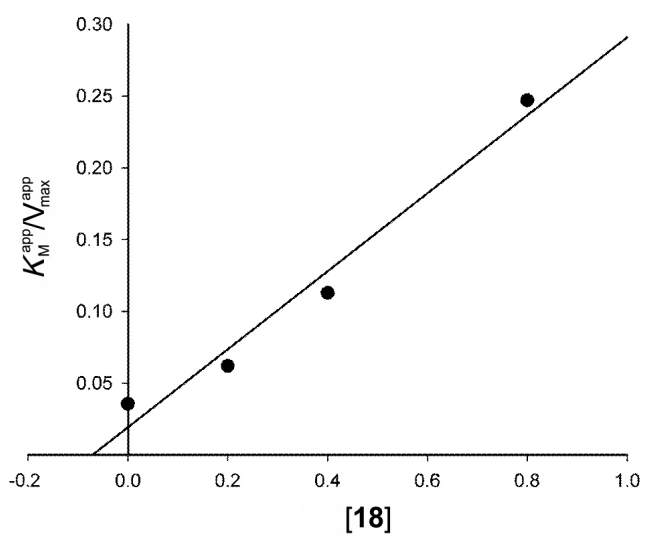

B

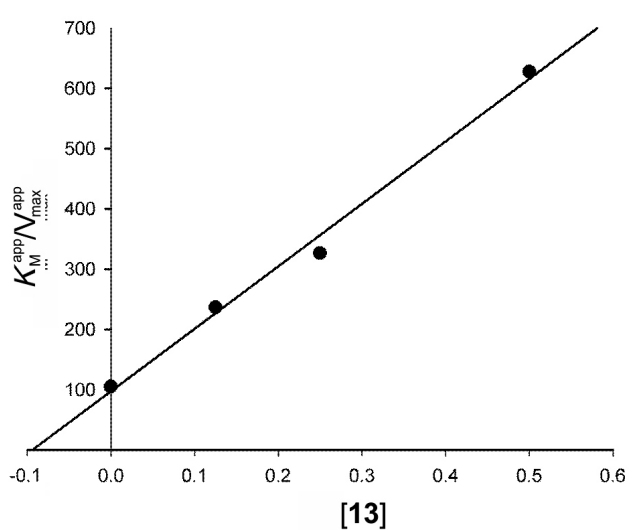

$\mathrm{D}$

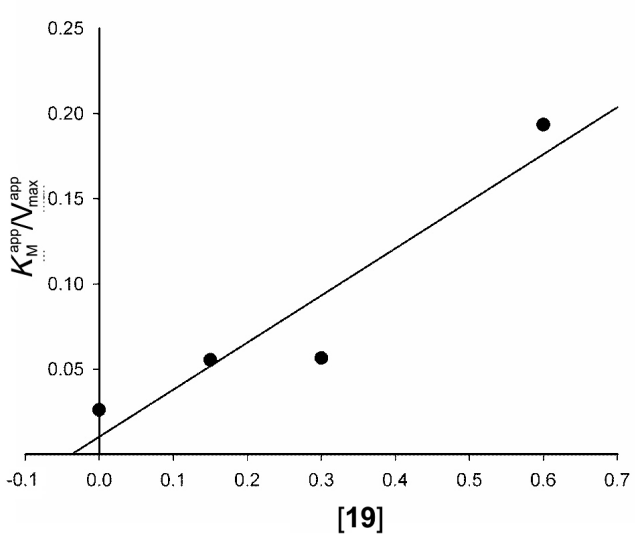

Figure 2. Determination of $K_{\mathrm{i}}$ values for a small series of SmPNP inhibitors. Kinetics assays were carried out in the same conditions described in Figure 1.

(obtained at three different inhibitor concentrations) converging at the $\mathrm{y}$-axis $\left(1 / \mathrm{V}_{\max }\right)$, whereas the slope $\left(K_{\mathrm{M}} / \mathrm{V}_{\max }\right)$ and the $\mathrm{x}$-axis intercepts $\left(-1 / K_{\mathrm{M}}\right)$ vary with inhibitor concentration (Figure 1). Consequently, the $\mathrm{V}_{\max }$ values remain constant, whereas the apparent values of $K_{\mathrm{M}}$ $\left(K_{\mathrm{M}}^{\text {app }}\right.$, defined as $\left.K_{\mathrm{M}}\left(1+[\mathrm{I}] / K_{\mathrm{i}}\right)\right)$ increase with increasing inhibitor concentrations. This behavior is consistent with a mutually exclusive binding mode between inhibitor and substrate, thus indicating that the inhibition of $S m$ PNP was found to be competitive for the inhibitors 7, 13, 18 and 19. A similar behavior was observed for the other compounds of the data set under the same experimental conditions (results not shown).

The determination of the inhibitor constant $K_{\mathrm{i}}$ is particularly relevant in the present case, since the $\mathrm{IC}_{50}$ values were obtained at only one concentration of substrate. For competitive inhibitors, $\mathrm{IC}_{50}$ values increase as the concentration of the substrate increases. As shown in Figure 2, the intersection on the $\mathrm{x}$-axis of the graphs of $K_{\mathrm{M}}$ app $/ \mathrm{V}_{\max }$ app versus inhibitor concentration indicates $-K_{\mathrm{i}}$. The $K_{\mathrm{i}}$ values are collected in Table 2 .

The data of Table 2 show that the values of $\mathrm{IC}_{50} / K_{\mathrm{i}}$ vary from 1.4 to 4.2 , which supports the competitive mechanism of inhibition. Despite the strong evidences that all of 9-deazaguanine inhibitors bind to the active site of the enzyme, the kinetic data does not provide insights into the binding profile of the inhibitors (in the substrate pocket of SmPNP). Therefore, crystallization and X-ray studies were carried out to complement the kinetic findings. Extensive soaking experiments afforded the crystallographic structure of $S m$ PNP in complex with the nanomolar inhibitor 7 (SmPNP-7) at $2.3 \AA$ (Figure 3 and Table 3).

As expected, the purine moiety shows a conserved H-bonding network to Glu203 and Asn245, in agreement with previous studies for the bovine and human PNPs. ${ }^{27-29}$ As can be seen in Figure 3, an extra H-bonding to a crystallographic water is found, although this feature could be also present in other 6-oxo purine inhibitors with moderate potency, such as compound $\mathbf{1 4}\left(\mathrm{IC}_{50}=22 \mu \mathrm{M}\right)$. As a consequence, the relative high potency of compound $7\left(\mathrm{IC}_{50}=0.82 \mu \mathrm{M}\right)$ might be a result of a more complex binding network that incorporates particular interactions of the 3-pyridine substituent at position 9 of the purine ring. Accordingly, this moiety not only H-bonds to Tyr202 and His 259, but also reaches a hydrophobic pocket lined by Met221 and Phe161. These experimental results led us to 
Table 3. Full data collection and refinement statistics for the SmPNP-7 crystallographic complex

\begin{tabular}{|c|c|}
\hline \multicolumn{2}{|c|}{ Data collection parameters } \\
\hline Space group & $\mathrm{P} 2{ }_{1} 2_{1} 2_{1}$ \\
\hline Cell dimensions / $\AA$ & $a=49.58 ; b=132.95 ; c=121.15$ \\
\hline Detector & MARMOSAIC 225 \\
\hline $\mathrm{X}$-ray source & LNLS MX2 \\
\hline Wavelength / $\AA$ & 1.43 \\
\hline Resolution range / $\AA$ & $27.61-2.30(2.42-2.30)$ \\
\hline Redundancy & $3.8(3.9)$ \\
\hline $\mathrm{R}_{\text {meas }} /(\%)$ & $11(56.7)$ \\
\hline $\mathrm{R}_{\mathrm{sym}} /(\%)$ & $9.3(48.6)$ \\
\hline Completeness / (\%) & $99.8(100.0)$ \\
\hline Total reflections & 671970 \\
\hline Unique reflections & 35710 \\
\hline $\mathrm{I} / \sigma \mathrm{I}$ & $11.4(3.4)$ \\
\hline \multicolumn{2}{|c|}{ Refinement parameters } \\
\hline $\mathrm{R} /(\%)$ & 20.4 \\
\hline Rfree / (\%) & 25.9 \\
\hline \multicolumn{2}{|l|}{ Ramachandran plot } \\
\hline Most favoured region / (\%) & 91.6 \\
\hline Residues in disallowed regions & 0.7 \\
\hline Overall B-factor (protein) & 36 \\
\hline Mean B-factor (ligand) & 61.6 \\
\hline No. protein atoms (one monomer) & 6332 \\
\hline No. water molecules & 348 \\
\hline No. ligand atoms & 76 \\
\hline r.m.s. bond lengths / $\AA$ & 0.008 \\
\hline r.m.s. bond angles / (deg.) & 1.063 \\
\hline
\end{tabular}

investigate the binding mode of other potent 9-deazaguanine inhibitors using different molecular docking strategies. In this context, FlexX and GOLD 4.12 were employed to cross-dock compound 7 into the SmPNP active site (PDB ID 1TD1). The best FlexX solution shows a RMSD of $1.29 \AA$ from the crystallographic coordinates of compound 7 (Figure 4A). Similar results were obtained with GOLD 4.12. These initial results prompted us to further explore the docking tools to provide additional SAR insights within this series.

The binding modes of the 9-deazaguanine derivatives shown in Figure 4B highlight the most important intermolecular interactions involved in ligand binding. The H-bonding pattern between the deazaguanine moiety and the side chains of Glu203 and Asn245 is fundamental, where the catalytic residue Asn245 donates a hydrogen bond to the carbonyl oxygen at the position 6 , and accepts a hydrogen bond from the $\mathrm{N}-\mathrm{H}$ group at position 7 of the purine ring. Similarly, the residue Glu203 accepts hydrogen bonds from the $\mathrm{NH}_{2}$ and $\mathrm{NH}$ groups at positions 2 and 1 , respectively. The $\pi-\pi$ interaction between the electron rich guanine ring and the side chain of Tyr202 might also be important for inhibitor binding. In addition, the side chain of Phe161 from the adjacent subunit generates a hydrophobic surface near the site entrance (Figure 4), which provides a suitable interaction region for hydrophobic moieties at position 9 of the purine ring, such as the para-phenyl and para-isopropyl benzyl of compounds $\mathbf{1 9}\left(\mathrm{IC}_{50}=0.1 \mu \mathrm{M}\right)$ and $\mathbf{1 8}\left(\mathrm{IC}_{50}=0.14 \mu \mathrm{M}\right)$, respectively. On the other hand, more bulk groups, such as the para-cresyl benzyl ether of compound $\mathbf{2 0}$ $\left(\mathrm{IC}_{50}=0.5 \mu \mathrm{M}\right)$, becomes more exposed to solvent and led a decrease in potency.
A

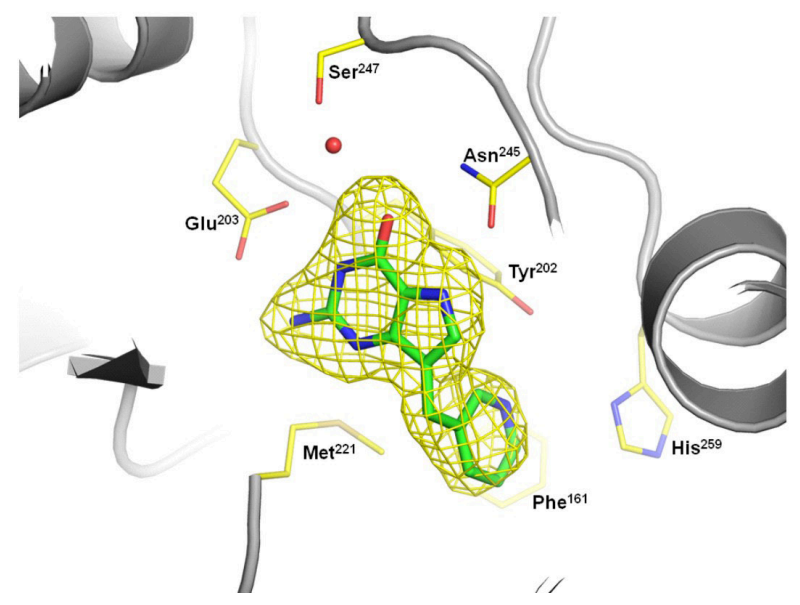

B

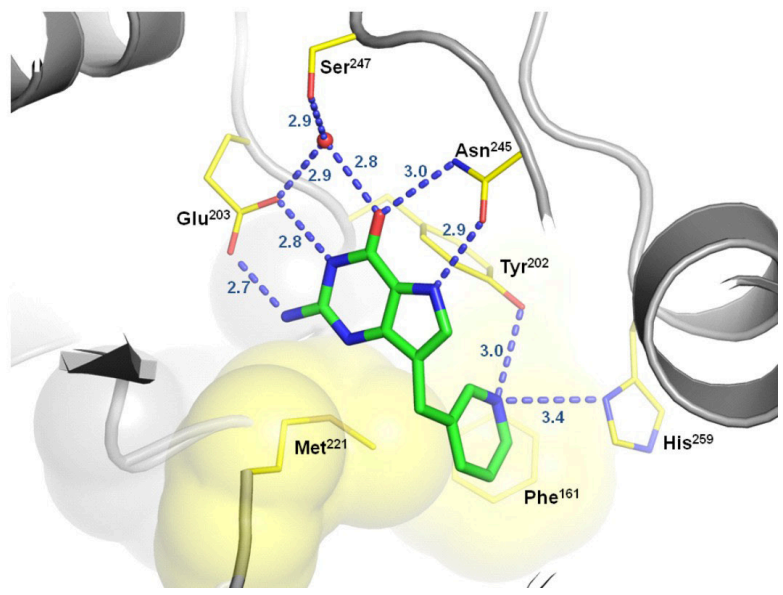

Figure 3. Crystallographic structure of compound 7 in complex with $S m$ PNP. A) Electron density (Fo-Fc map), contoured at 3.08, where the inhibitor was fit. B) Binding profile of compound 7 in the active site of $S m$ PNP. Selected residues from the active site are highlighted in yellow, whereas the protein structure is depicted in cartoon. Dashed lines indicate the H-bonding network of compound $\mathbf{7}$ in the active site of SmPNP. 

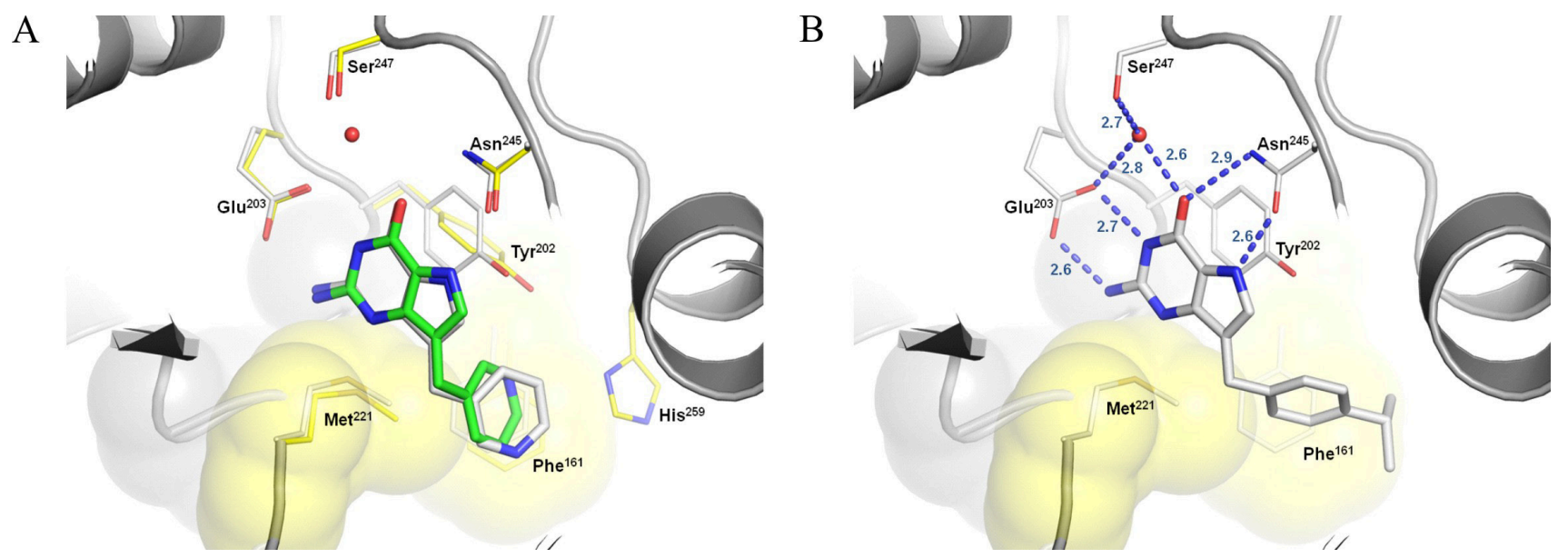

Figure 4. Proposed binding mode of $S m$ PNP inhibitors according to FlexX. A) Overlay of the best solution found for compound 7 onto its crystallographic pose (RMSD = 1.28 A). B) Interaction profile of compound 18 into the active site of SmPNP. Selected residues from the $S m$ PNP-7 complex (yellow) and SmPNP in complex with acetate (gray) is highlighted, and the dashed lines indicate the H-bonding network of compound 18.

\section{Conclusions}

The development of new therapeutic approaches to combat neglected tropical diseases continues to be one of the most important scientific and public health challenges facing humankind today. The identification of novel smallmolecule compounds that modulate specific biological targets is of great pharmaceutical interest, as this complex task requires the use of different drug design technologies. . $^{5,30}$ The integration of enzyme kinetics, structural analysis and molecular modeling studies provided important insights into the molecular basis underlying ligand binding affinity and SmPNP inhibition. The information gathered in this work should be useful in the design of new inhibitors having improved potency.

\section{Acknowledgments}

We gratefully acknowledge financial support from FAPESP (São Paulo Research Foundation), FAPESB (Fundação de Amparo à Pesquisa do Estado da Bahia) and CNPq (National Council for Scientific and Technological Development), Brazil. We are also grateful to BioCryst Pharmaceuticals, Inc. for the gift of the inhibitors employed in this work.

\section{References}

1. Hotez, P.; Molyneux, D. H.; Fenwich, A.; Kumaresan, J.; Sachs, S. E.; Sachs, J. D.; Savioli, L.; N. Engl. J. Med. 2007, $357,1018$.

2. Sabra, A. N.; Botros, S. S.; J. Parasitol. 2008, 94, 537.

3. Global Forum for Health Research; The 10/90 Report on Health Research, 2003-2004, Geneva, 2004. http://www.
globalforumhealth.org/Media-Publications/Publications/1090-Report-2003-2004

4. Guido, R. V. C.; Oliva, G.; Andricopulo, A. D.; Curr. Med. Chem. 2008, 15, 37.

5. Andricopulo, A. D.; Salum, L. B.; Abraham, D. J.; Curr. Top. Med. Chem. 2009, 9, 771; Cardoso, C. L.; Lima, V. V.; Zottis, A.; Oliva, G.; Andricopulo, A.; Wainer, I. W.; Moaddel, R.; Cass, Q. B.; J. Chromatogr., A 2006, 1120, 151.

6. Montgomery, J. A.; Med. Res. Rev. 1993, 13, 209.

7. Bzowska, A.; Kulikowska, E.; Shugar, D.; Pharmacol. Ther. 2000, 88, 349.

8. http://www.biocryst.com/clinical_pipeline, acessed in August 2010.

9. Pereira, H. M.; Cleasby, A.; Pena, S. D.; Franco, G. R.; Garratt, R. C.; Acta Crystallogr., Sect. D: Biol. Crystallogr. 2003, 59, 1096.

10. Kicska, G. A.; Tyler, P. C.; Evans, G. B.; Furneaux, R. H.; Schramm, V. L.; Kim, K. J.; Mol. Biol. 2002, 277, 3226.

11. Pereira, H. D.; Franco, G. R.; Cleasby, A.; Garratt, R. C.; J. Mol. Biol. 2005, 353, 584.

12. Shi, W.; Ting, L. M.; Kicska, G. A.; Lewandowicz, A.; Tyler, P. C.; Evans, G. B.; Furneaux, R. H.; Kim, K.; Almo, S. C.; Schramm, V. L.; J. Biol. Chem. 2004, 279, 18103.

13. Castilho, M. S.; Postigo, M. P.; Pereira, H. M.; Oliva, G.; Andricopulo, A. D.; Bioorg. Med. Chem. 2010, 18, 1421.

14. Postigo, M. P.; Guido, R. V. C.; Oliva, G.; Castilho, M. S.; Pitta, I. R.; Albuquerquer, J. F. C.; Andricopulo, A .D.; J. Chem. Inf. Model. 2010, 50, 1693.

15. Farutin, V.; Masterson, L.; Andricopulo, A. D.; Cheng, J.; Riley, B.; Hakimi, R.; Frazer, J. W.; Cordes, E. H.; J. Med. Chem. 1999, 42, 2422.

16. Andricopulo, A. D.; Yunes, R. A.; Chem. Pharm. Bull. 2001, $49,10$.

17. Kim, B. K.; Cha, S.; Parks, R. E., Jr.; J. Biol. Chem. 1968, 243 , 1771. 
18. Gohlke, H.; Hendlich, M.; Klebe, G.; J. Mol. Biol. 2000, 295, 337; http://pc1664.pharmazie.uni-marburg.de/drugscore/index.php, accessed in June 2009.

19. Leslie, A. G. W.; Acta Crystallogr., Sect. D: Biol. Crystallogr. 1999, 55, 1696.

20. Collaborative Computational Project, Number 4, The CCP4 suite: programs for protein crystallography; Acta Crystallogr., Sect. D 1994, 50, 760.

21. Vagin, A.; Teplyakov, A.; Acta Crystallogr. 2000, 56, 1622.

22. Murshudov, G. N.; Vagin, A.; Dodson, E. J.; Acta Crystallogr. 1997, 53, 240 .

23. Adams, P. D.; Grosse-Kunstleve, R. W.; Hung, L.; Ioerger, T. R.; McCoy, A. J.; Moriarty, N. W.; Read, R. J.; Sacchettini, J. C.; Sauter, N. K.; Terwilliger, T. C.; Acta Crystallogr. Sect D: Biol. Crystallogr. 2002, 58, 1948.

24. Emsley, P.; Cowtan, K.; Acta Crystallogr., Sect. D: Biol. Crystallogr. 2004, 60, 2126.

25. Brunger, A. T.; Acta Crystallogr., Section D: Biol. Crystallogr. 1993, 49, 24.

26. Laskowski, R. A.; Moss, D. S.; Thornton, J. M.; J. Mol. Biol. 1993, 231, 1049.

27. Castilho, M. S.; Postigo, M. P.; Paula, C. B. V.; Montanari, C. A.; Oliva, G.; Andricopulo, A. D.; Bioorg. Med. Chem. 2006, 14,516 .
28. Shewach, D. S.; Chern, J. W.; Pillote, K. E.; Townsend, L. B.; Daddona, P. E.; Cancer Res. 1986, 46, 519; Woo, P. W.; Kostlan, C. R.; Sircar, J. C.; Dong, M. K.; Gilbertsen, R. B.; J. Med. Chem. 1992, 35, 1451.

29. Secrist-3rd, J. A.; Niwas, S.; Rose, J. D.; Babu, Y. S.; Bugg, C. E.; Erion, M. D.; Guida, W. C.; Ealick, S. E.; Montgomery, J. A.; J. Med. Chem. 1993, 36, 1847.

30. Castilho, M. S.; Guido, R. V. C.; Andricopulo, A. D.; Bioorg. Med. Chem. 2007, 15, 6242; Andricopulo, A. D.; Yunes, R. A.; Nunes, R. J.; Savi, A. O. S.; Correa, R.; Cruz, A. B.; Cechinel, V.; Quim. Nova 1998, 21, 573; Salum, L. B.; Polikarpov, I.; Andricopulo, A. D.; J. Chem. Inf. Model. 2008, 48, 2243; Lima, E. O.; Queiroz, E. F.; Andricopulo, A. D.; Nunes, R. J.; Yunes, R. A.; Correa, R.; Cechinel, V.; Bol. Soc. Chil. Quim. 1999, 44, 185. Honorio, K. M.; Garratt R. C.; Polikatpov, I., Andricopulo, A. D.; J. Mol. Graphics Modell. 2007, 25, 921.

Submitted: September 10, 2010 Published online: December 21, 2010

FAPESP has sponsored the publication of this article. 